\title{
Evaluation of flaxseed effects on non-alcoholic fatty liver disease (NAFLD) in rabbits submitted to a hypercholesterolemic diet
}

\section{Caroline Tatim Saad ${ }^{1}$, Dalton Bertolim Précoma ${ }^{2}$, Alexandre Bueno Merlini ${ }^{3}$, Sérgio Ossamu Ioshii ${ }^{4}$, Ana Flávia Champoski ${ }^{5}$}

${ }^{1}$ State University of Ponta Grossa, Paraná, Brazil; ${ }^{2}$ Pontifical Catholic University of Paraná, Paraná, Brazil; ${ }^{3}$ Medical Student in State University of Ponta Grossa, Paraná, Brazil; ${ }^{4}$ Pontifical Catholic University of Paraná, Paraná, Brazil; ${ }^{5}$ Pontifical Catholic University of Paraná, Paraná, Brazil

*Corresponding author: Alexandre Bueno Merlini, $\mathrm{PhD}$, Department of Medicine, State University of Ponta Grossa. Av. Balduíno Taques, 1441, ap.43 Centro, 'Ponta Grossa, Paraná 84010-050, Brazil

Submission date: June 26, 2014; Acceptance date: October 27, 2014; Publication date: October 27, 2014

\begin{abstract}
Background: The aim of the present study is to evaluate the role of flaxseed in non-alcoholic fatty liver disease, as well as on the lipid profile in rabbits submitted to hypercholesterolemic diet.

Subject and Methods: 32 male rabbits, weighing approximately $1.5 \mathrm{~kg}$ and averaging four months of age, were distributed into three groups. Group 1 received standard food plus $0.5 \%$ of cholesterol from dried egg, during 8 weeks. Group 2 obtained the same diet in the first 4 weeks, and $8 \mathrm{mg} / \mathrm{kg}$ of ground flaxseed was added in the remaining weeks. Lastly, group 3 was fed with the previous group's increased diet throughout the entire period. In the follow-up, the animals were euthanized, and liver blades were prepared to evaluate the histopathologic study. The evaluation score of NAFLD (ESN), as well as plasma levels of total cholesterol, LDLcholesterol, HDL-cholesterol, triglycerides and body weight, were all determined.
\end{abstract}

Results: Increased levels of total cholesterol were obtained in both groups, with the smallest variation found in G3 ( $\mathrm{p}=0.002)$. This variation was also found when the levels of LDLcholesterol were assessed $(\mathrm{p}=0.001)$. There was a reduction of triglyceride levels at the end of the study in G3 ( $p=0.008)$. A variation was noticed between the ESN groups, but the induced reduction was not statistically significant.

Conclusion: Further studies are necessary, in order to elucidate the effects of flaxseed in NAFLD as well as in diseases that have risk factors for the development of the disease. 
Keywords: non-alcoholic fatty liver disease, flaxseed, experimental study, functional food

\section{BACKGROUND}

A distinct group of liver diseases is related to metabolism disorders, either acquired or inherited. The non-alcoholic fatty liver disease (NAFLD) is the most common acquired metabolic disorder and it is characterized by the presence of hepatic steatosis in individuals who do not consume alcohol, or do so in very small quantities (less than $20 \mathrm{~g}$ of ethanol per day) [1-3]. The understanding of the epidemiology and natural history of NAFLD has increased; it is the most common form of chronic liver disease in the Western world, and it's becoming more important in other parts of the world [4].

NAFLD includes simple hepatic steatosis, steatosis accompanied by minor, non-specific inflammation, and non-alcoholic steatohepatitis (NASH) [1]. Bland steatosis remains stable for a number of years and will probably never progress, in many cases. In contrast, NASH is a condition in which there is hepatocyte injury that may progress to cirrhosis in $10 \%$ to $20 \%$ of cases. The main components of NASH are hepatocyte ballooning, lobular inflammation, and steatosis. With the progress of the disease, fibrosis occurs [5].

$\mathrm{NASH}$ affects men and women equally, and the condition is strongly associated with obesity and other components of the metabolic syndrome (MS), such as dyslipidemia, hyperinsulinemia, and insulin resistance. It is estimated that more than $70 \%$ of obese individuals have some form of NAFLD, and the epidemic of obesity heightens the concern that it will increase in prevalence $[4,6]$.

Because of the association between NASH and the MS, cardiovascular disease is a frequent cause of death in patients with NASH. The goal of treating individuals with NASH is to reverse the steatosis and prevent cirrhosis [7]. The current management strategy seeks to correct the underlying risk factors, such as obesity and hyperlipidemia, and to treat insulin resistance [8].

Clinical trials and experimental models have shown that the inclusion of functional foods in the diet promotes improvements in risk factors linked to cardiovascular diseases, such as hypercholesterolemia [9]. Currently, many foods with functional properties have been considered as a prevention method for atherosclerotic lesions and other cardiovascular diseases, mainly because their compositions contain, for example, high percentages of n-3 fatty acids, plant sterol esters, and vegetable fiber [10,11].

The flaxseed (Linum usitatissimun L.) has been considered a functional ingredient of great importance by the American Heart Association. The importance is due to its functional combinations, such as linolenic acid, lignans, and soluble fibers that are potentially beneficial to our health, mainly to the heart [12].

The active component of flaxseed (lignans - secoisolariciresinol diglucoside - SDG) has significant antioxidant effects resulting from the inhibition of the DNA's divisions and lipid peroxidation, as well as a decreased production of reactive oxygen species. Several clinical and experimental trials provide evidence of the numerous cardioprotective effects of lignans [3].

Since NASH is strongly associated with metabolic syndrome, as well as cardiovascular diseases, the study of functional properties of flaxseed is of extreme importance and enrichment for the scientific community and the population [7]. 


\section{SUBJECT AND METHODS}

Thirty-two white adult male rabbits (New Zealand), averaging four months of age, were selected for this study. Animals were handled in compliance with the Guiding Principles in the Care and Use of Animals, and protocol approval was obtained from the Pontifical Catholic University Animal Research Committee. The animals were divided into three groups, for a period of eight weeks: group I (GI), the control group, 11 rabbits; group II (GII), 10 rabbits, fed throughout half of the period with flaxseed; group III (GIII), 11 rabbits, fed throughout the entire study with flaxseed. During the 56-day study, the animals from GI were fed with a specific diet (Nuvilab®), plus $1 \%$ cholesterol from lyophilized egg. This diet (Nuvilab®) does not alter the lipid metabolism of the animals. GII received, from the fifth week onwards, besides the standard diet, $8 \mathrm{mg} / \mathrm{kg}$ of flaxseed. And GIII was fed with GII's second diet for the entire the period. On day 56, the animals were submitted to liver resection. Anesthesia was induced with ketamine (Vetarnacol®, König: 30mg/kg) and intramuscular xylazine (Coopazine ${ }^{\circledR}$; Coopers: 6mg/kg). After the procedure, the rabbits were sacrificed via a lethal barbiturate dosage.

\section{Blood chemistry}

Blood samples were collected on the first day of the experiment, and immediately before sacrifice via cardiac puncture. The laboratory assessment included: total cholesterol (TC), highdensity lipoprotein cholesterol (HDL-C), low-density protein cholesterol (LDL-C), and triglycerides (TGL). Measurements were taken using an automated system (Abbott Architect ci8200; Abbott Laboratories, Abbott Park, II).

\section{Histological Analysis}

The liver segments were removed and fixed with $10 \%$ formaldehyde, buffered with phosphate $(\mathrm{pH}=7.6)$, and then embedded with paraffin. Three slices with two histological sections were prepared, one with a left medial lobe liver sample, and one with a quadrate lobe liver sample. One slice was stained with hematoxylin and eosin (HE), and two slices were stained with periodic acid Schiff (PAS) and Gomori's trichrome. The slices were analyzed blindly for steatosis, lobe inflammation, hepatic cell balonization, and fibrosis status according to the histological score system for NAFLD, using a five-head Olympus® BX 40 microscope.

\section{Statistical Analysis}

Categorical variables were expressed as percentages, and continuous variables were expressed as an average \pm standard deviation (SD). The Shapiro-Wilks test was used for testing sample normality. For quantitative parameters, the student t-test and Mann-Whitney nonparametric test were used for comparison between groups. Statistical significance was indicated by a value of $p$ $<0.05$. For multiple comparisons through Fisher's exact test the level of significance was corrected by Bonferroni. Therefore, for these comparisons, statistical significance was indicated by a value of $p<0.017$. Analyses were performed using Statistica/W version 5.1 (StatSoft, Tulsa, OK).

\section{RESULTS}

Metabolic and lipid profiles 
Animal weight gain did not differ among the groups between baseline and euthanasia (Table 1), However, when individually analyzed, there was a significant difference between the basal weight and euthanasia's weight $(\mathrm{p}<0,001)$. In relation to the lipid profile, a significant difference between the groups was found when analyzing the total cholesterol, LDL-cholesterol and HDLcholesterol (Table 2).

Table 1. Weight variation between groups

\begin{tabular}{|ccccc|}
\hline Weight & Group & $\boldsymbol{n}$ & Mean \pm & $\boldsymbol{p}$ \\
\hline Basal (B) & SI & 11 & $1882 \pm 267$ & \\
& GII & 10 & $1876 \pm 209$ & 0,268 \\
& GIII & 11 & $2053 \pm 345$ & \\
\hline Euthanasia (E) & GI & 11 & $2975 \pm 165$ & 0,367 \\
& GII & 10 & $3154 \pm 268$ & \\
\hline Difference (E-B) & GIII & 11 & $3080 \pm 380$ & 0,071 \\
& GI & 11 & $1120 \pm 277$ & \\
\hline
\end{tabular}

Table 2. Lipid profile at baseline (T0) and euthanasia (T8)

\begin{tabular}{|c|c|c|c|c|c|c|c|}
\hline Variable & $\begin{array}{c}\mathbf{G} 1-\mathbf{T}_{\mathbf{0}} \\
\text { Mean } \pm \mathbf{S D}\end{array}$ & $\begin{array}{c}\text { G1-T } \\
\text { Mean } \pm \text { SD }\end{array}$ & $\begin{array}{c}\mathbf{G 2}-\mathbf{T}_{\mathbf{0}} \\
\text { Mean } \pm \text { SD }\end{array}$ & $\begin{array}{c}\mathbf{G 2}-\mathbf{T}_{\mathbf{8}} \\
\text { Mean } \pm \mathbf{S D}\end{array}$ & $\begin{array}{c}\mathbf{G 3}-\mathbf{T}_{\mathbf{0}} \\
\text { Mean } \pm \mathbf{S D}\end{array}$ & $\begin{array}{c}\mathbf{G 3}-\mathbf{T}_{\mathbf{8}} \\
\text { Mean } \pm \text { SD }\end{array}$ & $\begin{array}{c}p \text { value } \\
\text { G1xG2xG3 }\end{array}$ \\
\hline $\begin{array}{c}\text { Total } \\
\text { cholesterol }\end{array}$ & $75,8 \pm 36,7$ & $\begin{array}{c}642,3 \pm \\
392,4 \\
\\
\text { Variation } \\
\text { G1 } \\
566,5 \pm \\
372,7\end{array}$ & $73,5 \pm 25,3$ & $\begin{array}{c}731,6 \pm \\
258,9 \\
\\
\text { Variation } \\
\text { G2 } \\
658,1 \pm \\
250,4\end{array}$ & $61,7 \pm 14,9$ & $\begin{array}{c}321,7 \pm \\
215,3 \\
\text { Variation } \\
\text { G3 } \\
260 \pm 208,6\end{array}$ & $\begin{array}{l}\text { T0: } 0,442 \\
\text { T8: } \mathbf{0 , 0 0 9}\end{array}$ \\
\hline $\begin{array}{c}\text { LDL- } \\
\text { cholesterol }\end{array}$ & $34,8 \pm 33,7$ & $\begin{array}{c}605,4 \pm \\
390,1 \\
\text { Variation } \\
\text { G1 } \\
570,6 \pm \\
370,2\end{array}$ & $30,6 \pm 21,7$ & $\begin{array}{c}694,8 \pm \\
255,1 \\
\text { Variation } \\
\text { G2 } \\
664,2 \pm 246\end{array}$ & $12,8 \pm 6,6$ & $\begin{array}{c}294 \pm 211,4 \\
\text { Variation } \\
\text { G3 } \\
281,1 \pm \\
206,9\end{array}$ & $\begin{array}{c}\text { T0:0,085 } \\
\text { T8:0,01 }\end{array}$ \\
\hline $\begin{array}{c}\text { HDL- } \\
\text { cholesterol }\end{array}$ & $21,3 \pm 9,3$ & $\begin{array}{l}23,7 \pm 8,8 \\
\text { Variation } \\
\text { G1 } \\
2,5 \pm 11,7\end{array}$ & $20,3 \pm 4,7$ & $\begin{array}{c}19,1 \pm 4,6 \\
\text { Variation } \\
\text { G2 } \\
-1,2 \pm 7,8\end{array}$ & $24,4 \pm 5,8$ & $\begin{array}{c}15 \pm 3,8 \\
\text { Variation } \\
\text { G3 } \\
-9,4 \pm 6,6\end{array}$ & $\begin{array}{c}\text { T0:0,0381 } \\
\text { T8:0,01 } \\
\\
\mathbf{0 , 0 1 4}\end{array}$ \\
\hline Triglycerides & $98,5 \pm 40,6$ & $\begin{array}{c}65,6 \pm 26,2 \\
\text { Variation } \\
\text { G1 } \\
-32,8 \pm 48,8\end{array}$ & $112,2 \pm 31,2$ & $\begin{array}{c}88,5 \pm 37,3 \\
\text { Variation } \\
\text { G2 } \\
-23,7 \pm 45,4\end{array}$ & $161 \pm 88,1$ & $\begin{array}{c}63,8 \pm 31,9 \\
\text { Variation } \\
\text { G3 } \\
-97,2 \pm 98,3\end{array}$ & $\begin{array}{c}\text { T0:0,050 } \\
\text { T8:0,166 } \\
0,166\end{array}$ \\
\hline
\end{tabular}




\section{Histological Analysis}

The non-alcoholic fatty liver disease was successfully induced in all groups. However, there was no statistically significant difference between them. The frequency of the histologic findings is showed in Table 3.

Table 3. Results of histological analysis

\begin{tabular}{|c|c|c|c|c|c|}
\hline Item & Definition & $\begin{array}{l}\mathrm{S} \\
\text { core }\end{array}$ & G1 & $\mathrm{G} 2$ & G3 \\
\hline Steatosis grade & $\begin{array}{c}<5 \% \\
5 \text { to } 33 \% \\
33 \text { to } 66 \% \\
>66 \%\end{array}$ & $\begin{array}{l}0 \\
1 \\
2 \\
3\end{array}$ & $\begin{array}{c}27,27 \% \\
63,64 \% \\
9,09 \% \\
0\end{array}$ & $\begin{array}{c}10 \% \\
70 \% \\
20 \% \\
0\end{array}$ & $\begin{array}{c}36,36 \% \\
63,64 \% \\
0 \\
0\end{array}$ \\
\hline Fibrosis stage & $\begin{array}{c}\text { None } \\
\text { Perisinusoidal or } \\
\text { periportal }\end{array}$ & $\begin{array}{l}0 \\
1\end{array}$ & $\begin{array}{l}81,82 \% \\
18,18 \%\end{array}$ & $\begin{array}{c}100 \% \\
0\end{array}$ & $\begin{array}{l}81,82 \% \\
18,18 \%\end{array}$ \\
\hline $\begin{array}{c}\text { Lobular } \\
\text { inflammation }\end{array}$ & $\begin{array}{c}\text { No foci } \\
<2 \text { foci per } 200 x \text { field } \\
2-4 \text { foci per } 200 x \\
\text { field } \\
>4 \text { foci per } 200 x \text { field }\end{array}$ & $\begin{array}{l}0 \\
1 \\
2 \\
3\end{array}$ & $\begin{array}{c}90,91 \% \\
9,09 \% \\
0 \\
0\end{array}$ & $\begin{array}{c}70 \% \\
30 \% \\
0 \\
0\end{array}$ & $\begin{array}{c}72,73 \% \\
27,27 \% \\
0 \\
0\end{array}$ \\
\hline $\begin{array}{c}\text { Hepatocellular } \\
\text { ballooning }\end{array}$ & $\begin{array}{c}\text { None } \\
\text { Few balloon cells } \\
\text { Many cells/prominent } \\
\text { ballooning }\end{array}$ & $\begin{array}{l}0 \\
1 \\
2\end{array}$ & $\begin{array}{c}0 \\
72,73 \% \\
27,27 \%\end{array}$ & $\begin{array}{c}0 \\
60 \% \\
40 \%\end{array}$ & $\begin{array}{c}0 \\
36,36 \% \\
63,64 \%\end{array}$ \\
\hline
\end{tabular}

\section{DISCUSSION}

NAFLD is now the most common liver disease in the United States, and possibly worldwide. Furthermore, the number of affected patients is growing rapidly, and the disease has reached epidemic proportions. It is now believed to be the hepatic manifestation of the metabolic syndrome (MS), and an independent predictor of cardiovascular morbidity and mortality [4].

Although not every variant of NAFLD is associated with insulin resistance and the MS, its main component, the NASH, is now considered a clinical entity with characteristics that allow defining it as a disease [7]. Its pathogenesis, although not yet fully elucidated, includes the accumulation of excess lipid in hepatocytes by insulin resistance, and the oxidative stress causing lipid peroxidation, which activates pro-inflammatory cytokines [1].

There is still no specific pharmacological treatment for NAFLD or NASH. Most drugs are used to mitigate some of the symptoms or treat comorbidities, such as those present in the MS (obesity, insulin resistance or DM2, hypertension, dyslipidemias - particularly hypertriglyceridemia). The therapeutic options currently employed improve upon the laboratory abnormalities associated with NAFLD, but it proved ineffective in reducing the degree of steatosis, perhaps due to the lack of randomized controlled trials (RCT's) [9].

Flaxseed is considered to be one of the richest sources of $\Omega-3 \alpha$-linolenic fatty acids, and therefore the richest source of mammalian lignan precursors such as secoisolariciresinol 
diglucoside (SDG), pinoresinol, matairesinol, and lariciresinol, among others. Due to the antioxidant, anti-proliferative, anti-estrogenic, anti-aromatase and anti-angiogenic activities, its effect on various diseases has been studied, including on liver diseases such as NAFLD [12,13].

In previous studies, we found that SDG reduces the high lipid levels in rats, which was induced through a hypercholesterolemic diet. In a clinical trial, Fukumitsu et al. concluded that when compared to a placebo, the administration of $100 \mathrm{mg}$ of SDG resulted in a significant reduction of the LDL/HLD cholesterol ratio, and of the levels of alanine aminotransferase and gamma-glutamyl-transpeptidade, these being typically increased in fractions in NAFLD [14].

In this study we observed that there was a marked increase in total cholesterol levels throughout all groups, and in the G3 (diet with flaxseed supplementation since the beginning), this variation was small compared to other groups. This same observation was made in relation to LDL.

In a double-blind, randomized, controlled study, guided by Wu et al., it was noted that 12week supplementation with flaxseed, within a program of changing styles and habits of life, significantly reduced the number of MS's components, including weight, waist circumference, total cholesterol, LDL cholesterol, apolipoproteins B and E, and arterial pressure [15].

Regarding HDL, there was no significant change between groups, but we observed an HDL decrease in in $\mathrm{G} 3$ at the end of the study. A clinical trial organized by Bloedon and colleagues found that, in males, there was a $16 \%$ reduction in HDL-a follow-up of cholesterol in 5 weeks, and $9 \%$ when measured in 10 weeks [16].

When we analyzed the triglycerides, we found a significant reduction in levels of G3.

The role of the flaxseed in triglyceride levels, according to the literature, is controversial. Some studies, such as a meta-analysis by Pan et al., did not find significant changes in plasma levels of TG.84. Meanwhile, a research with a follow-up of 64 weeks in birds, led by Cherian et al., said that a diet with $10 \%$ of flaxseed significantly decreased hepatic and plasma levels of triglycerides [17].

The NAFLD activity score (DAS), which corresponds to the unweighted sum of scores of steatosis, lobular inflammation and hepatocyte ballooning, is appropriately related with the presence of NASH when $\geq 5$, with the absence when $\leq 2$, and had no value discriminating between 3 and 4 in the study for its validation.

Although there was a variation between groups of DAS as well as the individual components of the DAS, this reduction was not statistically significant, showing that flaxseed was not able to reduce the degree of steatosis in rabbits submitted to hypercholesterolemic diet to $0.5 \%$ dried egg. This may be due to sample size, even with the duration of the study.

The data in this study showed positive effects of flaxseed in the correction of lipid disorders associated with NAFLD, but was not effective in reducing hepatic steatosis. Human studies show that the improvement in steatosis is associated with a previous improvement of lipid disorders and other disorders associated with MS, as well as reducing conditions that lead to oxidative stress. This shows the need for longer studies and larger samples.

In the study by Cherian et al., it was noted that the intervention with flaxseed reduced the plasma and hepatic lipid content, the accumulation of triglycerides in the liver (steatosis), the total number of lipid vacuoles, and the number of hepatocytes with $75 \%$ or more lipid 
infiltration. However, as already mentioned, the follow-up study took 64 weeks, and this may be the cause of the difference in results [18].

In other studies, such as Lirussi et al., the assessment of liver function showed an increased activity of gamma-glutamyl transpeptidase, but no change in aminotransferases. These studies affirm the potential of flaxseed as a protector of the progression of liver damage, but not as treatment of DHGNA [19].

There are not many studies analyzing NAFLD in rabbits. In this study, the model used followed the line of the supervisor, and may contribute to the development of a standard model of NAFLD in experimental rabbits. Given the biochemical, hematological and histopathological results obtained, we can think about a correlation of applications of flaxseed in humans, since the pathogenesis and mechanism of inflammatory injury in the two species are the same. However, very few studies reported this association in humans, and most of them using rodents, due to the ease of production.

Among those who already carried out similar studies, we have Sturzeneker et al., who evaluated the role of olmesartan, an angiotensin receptor blocker-II, in the course of NAFLD. The results showed that, after NAFLD being installed, this drug prevents the development of fibrosis and lobular inflammation, and reduced the risk of NASH. However, the drug was not effective in preventing steatosis [20].

Another study by Prim et al. found that the intake of flaxseed lowered LDL-cholesterol and total cholesterol. However, there was no significant difference in the presence or degree of atherosclerotic lesions in the group that received the seed and the group that did not [21].

\section{CONCLUSION}

According to what was seen, when we compare the results obtained in this study with those found in medical literature, we find that they are similar, since both managed to obtain an improvement in lipid profile (triglycerides, total cholesterol and LDL-cholesterol) and to prevent the progression of disease nonalcoholic fatty liver.

Further studies are necessary, in order to elucidate the effects of flaxseed in NAFLD as well as in diseases that have risk factors for the development of the disease.

\section{Competing interests:}

All the authors declare no conflict of interest.

\section{Authors Contributions:}

All the authors contributed to this study.

\section{Abbreviations:}

NAFLD, non-alcoholic fatty liver disease; NASH, non-alcoholic steatohepatitis; MS, metabolic syndrome; SDG, secoisolariciresinol diglucoside; CT, total cholesterol; HDL-C, high-density lipoprotein cholesterol; LDL-C, low-density lipoprotein cholesterol; TGL, triglycerides; SD, standard deviation; DM, diabetes mellitus;

\section{Acknowledgements and Funding:}


We thank the Postgraduate Program in Surgery of Pontifical Catholic University of Paraná, to which the present study was bound.

\section{REFERENCES}

1. Basaranoglu M; Kayacetin S; Yilmaz N; Kayacetin E; Tarcin O; Sonsuz A. Understanding mechanisms of the pathogenesis of non-alcoholic fatty liver disease. World J Gastroenterol. 2010; 16(18):2223-6.

2. Volzke H. Multicausality in fatty liver disease: is there a rationale to distinguish between alcoholic and non-alcoholic origin? World J Gastroenterol. 2012; 18(27):3492-501.

3. Cerovic I; Mladenovic D; Jesic R; Naumovic T; Brankovic M; Vucevic D et al. Alcoholic liver disease/not-alcoholic fatty liver disease index: distinguish alcoholic from non-alcoholic fatty liver disease. Eur J Gastroenterol Hepatol. 2013; 25(8):899904.

4. Cauchy F; Fuks D; Zarzavadjian Le Bian A; Belghiti J; Costi R. Metabolic syndrome and non-alcoholic fatty liver disease in liver surgery: the new scourges? World $\mathrm{J}$ Hepatol 2014; 6(5):306-14.

5. Toshikuni N; Tsutsumi M; Arisawa T. Clinical differences between alcoholic liver disease and non-alcoholic fatty liver disease. World J Gastroenterol. 2014; 20(26):8393-406.

6. Chalasani N; Younossi Z; Lavine JE; Diehl AM; Brunt EM; Cusi K et al. The diagnosis and management of non-alcoholic fatty liver disease: Practice guideline by the American Association for the Study of Liver Diseases, American College of Gastroenterology, and the American Gastroenterological Association. Am J Gastroenterol. 2012; 107(6):811-26.

7. Brea A, Puzo J. Non-alcoholic fatty liver disease and cardiovascular risk. Int J Cardiol. 2013; 167(4):1109-17.

8. Sourianarayanane A, Pagadala MR, Kirwan JP. Management of non-alcoholic fatty liver disease. Minerva Gastroenterol Dietol. 59(1):69-87.

9. Beaton MD. Current treatment options for non-alcoholic fatty liver disease and nonalcoholic steatohepatitis. Can J Gastroenterol. 2012; 26(6):353-7.

10. Elliott C; Frith J; Day CP; Jones DE; Newton JL. Functional impairment in alcoholic fatty liver disease and non-alcoholic fatty liver disease is significant and persists over 3 years of follow-up. Dig Dis Sci 2013; 58(8):2883-91.

11. Colak Y; Tuncer I; Senates E; Ozturk O; Doganay L; Yilmaz Y. Non-alcoholic fatty liver disease: a nutritional approach. Metab Syndr Relat Disord. 2012; 10(3):161-6.

12. Prasad K, Mantha SV, Munir AD. et al. Reduction of hypercholesterolemic atherosclerosis by CDC - flaxseed with very low alpha - lonolenic acid. Atherosclerosis 136:367-75, 1998.

13. Prasad K. Antihypertensive activity of secoisolariciresinol diglucoside isolated from flaxseed: role of guanylate cyclase. Int J Angiol 13:7-14, 2004. 
14. Fukumitsu S, Aida K, Shimizu H, Toyoda K. Flaxseed lignan lowers blood cholesterol and decreases liver disease risk factors in moderately hipercholesterolemic men. Nut Res. 2010; 30(7):441-6.

15. Wu H, Pan A, Yu Z, Qi Q, Lu L, Zhang G et al. Lifestyle counseling and supplementation with flaxseed or walnuts influence the management of metabolic syndrome. J Nutr. 2010; 140(11):1937-42.

16. Bloedon LT, Balikai S, Chittams J, Cunnane SC, Berlin JA, Rader DJ et al. Flaxseed and cardiovascular risk factors: results from a double blind, randomized, controlled clinical trial. J Am Coll Nutr. 2008; 27(1):65-74.

17. Pan A, Yu D, Demark-Wahnefried W, Franco OH, Lin X. Meta-analysis of the effects of flaxseed interventions on bloods lipids. Am J Clin Nutr. 2009; 90(2):288-97.

18. Cherian G, Hayat Z. Long-term effects of feeding flaxseeds on hepatic lipid characteristics and histopathology of laying hens. Poult Sci. 2009; 88(12):2555-61.

19. Lirussi F, Azzalini L, Orando S, Orlando R, Angelico F. Antioxidant supplements for non-alcoholic fatty liver disease and/or steatohepatitis. Cochrane Database Syst Rev. 2007

20. Sturzeneker MC, Ioshii SO, Villela Baroncini LA, Précoma DB. Olmesartan severely weakened the development of NASH in an animal model of hypercholesterolemia. Atherosclerosis. 2011; 216(1):97-102.

21. Prim CR, Baroncini LA, Précoma LB, Caron PH, Winter G, Poletti MO, Précoma DB. Effects of linseed consumption for a short period of time on lipid profile and atherosclerotic lesions in rabbits fed a hypercholesterolaemic diet. Br J Nutr. 2012; 107(5):660-4. 